\title{
Uma Nova Metodologia para Formação de Grupos em VANETs
}

\author{
André R. Vieira e Claudio M. Farias \\ PPGI - Universidade Federal do Rio de Janeiro \\ \{arvieira,cmfarias\}@ labnet.nce.ufrj.br
}

\author{
Wilson S. Melo Jr. \\ Inmetro - Duque de Caxias - RJ \\ wsjunior@inmetro.gov.br
}

\begin{abstract}
Resumo-This work proposes a new methodology to create groups in intervehicular networks as a basis for complex applications involving smart vehicles. We proposed a distributed protocol whose purpose is to build a higher number of groups in less time, keeping the vehicles grouped for most of their journey. We implement our proposal in two versions. The first one considers only the interaction among vehicles. The second includes a group merge functionality. We validate both versions of our protocol using simulation with real traffic data. We evaluate the amount of created groups and their persistence and size.

Index Terms-VANET, redes veiculares, clustering, agrupa-
\end{abstract} mento de veículos, algoritmo distribuído

\section{INTRODUÇÃO}

Veículos inteligentes ou autônomos têm sido foco de diversos estudos na última década. Em grande parte, isso se deve ao grande potencial de ganhos como otimização da fluidez de tráfego, redução na emissão de poluentes, maior segurança e proteção para motoristas, passageiros e pedestres, e melhor gerenciamento dos modais de transporte urbano [1]. Trabalhos recentes reportam avanços em diversas áreas relacionadas ao tema, tais como tecnologias de sensoriamento, atuadores, unidades de bordo (do inglês, On Board Unit ou OBUs), redes veiculares, entre outras [2], [3]. No contexto da comunicação interveicular, esta necessita atender requisitos inerentes à mobilidade dos nós da rede [1]. Mudanças rápidas na configuração da topologia de rede, interferência de obstáculos externos (e.g., construções ou obras de interdição ao longo das vias), desvanecimento e necessidade de respostas em tempo real são alguns dos desafios encontrados. Diante deles, pesquisadores buscam por soluções de problemas complexos e de natureza distribuída, como por exemplo a disputa do direito de passagem para veículos e o gerenciamento e condução de comboios autônomos [4], [5], [6], [7], [8]. Esses esforços demandam muitas vezes a simplificação do problema, sendo que a formação de grupos de veículos constitui uma estratégia eficiente para a otimização de troca de mensagens [9].

A literatura apresenta diferentes cenários e abordagens associadas à formação de grupo de veículos. Entre os primeiros trabalhos sobre o tema é possível encontrar abordagens baseadas em grafos [5], com formação de grupos também por intervalos de velocidade [4]. Trabalhos mais recentes propõem a criação de grupos para tratar problemas como o gerenciamento de comboios [6], decisão quanto ao direito de passagem em cruzamentos [7], [8] e a otimização de redes ponto a ponto para levar conectividade a locais de desastre [10]. A maioria desses trabalhos aborda os problemas citados propondo soluções que tratam desafios da formação de grupos em Vehicular AdHoc Networks (VANETs). Dentre esses desafios, tem-se a seleção do líder, a afiliação de membros, gerenciamento do grupo, robustez, e validação de algoritmos e protocolos usando modelagem e simulação [9]. As abordagens propostas são normalmente voltadas para um problema específico. Além disso, a validação das metodologias existentes raramente considera testes em ambientes reais de tráfego, ou simulações envolvendo dados reais. Esse problema constitui um desafio de complexidade não desprezível, uma vez que ferramentas de simulação envolvendo tanto aspectos de rede quanto de mobilidade de tráfego não proveem um mecanismo completo para reprodução da diversidade de situações que podem ser encontradas no mundo real [11]. Existe ainda a dificuldade encontrada na comparação de soluções propostas na literatura. Isso se deve à grande quantidade de simuladores utilizados, aos diferentes tipos de modelos de canais de comunicação e à diversidade de cenários possíveis [9].

$\mathrm{O}$ presente trabalho se diferencia ao propor uma metodologia que atende de forma geral a demanda de formação grupos em VANETs. O objetivo é obter uma solução-base para aplicações que requerem o tratamento rápido de um conjunto significativo de mensagens, minimizando o tempo em que os veículos trafegam sozinhos. Ele apresenta três contribuições principais:

- Uma nova metodologia que prioriza a geração do maior número de grupos de veículos, ao mesmo tempo que favorece o agrupamento célere, maximizando o tempo de permanência dos veículos em um grupo.

- Um protocolo distribuído, onde os veículos criam e dissolvem grupos de forma independente, sem a necessidade de uma coordenação central.

- Uma validação usando a ferramenta de simulação VSimRTI [12], alimentada por dados reais de tráfego disponibilizados pela Companhia de Engenharia de Tráfego do Rio de Janeiro [13].

As próximas seções detalham essas contribuições, sendo que a seção II aborda os trabalhos relacionados ao tema, a seção III apresenta a proposta de metodologia e descreve nosso protocolo distribuído de geração de grupos, a seção IV descreve os procedimentos de simulação e os resultados obtidos, e por fim 
a seção $\mathrm{V}$ apresenta as conclusões e trabalhos futuros.

\section{TRABALHOS RELACIONADOS}

O agrupamento de veículos em redes veiculares tem sido estudado nas últimas duas décadas, com trabalhos iniciais [14], [4], [5] que introduzem aspectos revisitados em trabalhos mais recentes [15], [6], [10], [9], [7], [8]. Dentre os problemas tratados, destacam-se (i) o uso da rede veicular para troca de informações e entretenimento; (ii) a simplificação de problemas clássicos, como a disputa do direito de passagem em cruzamentos ou a coordenação de um comboio de veículos; e (iii) a otimização de roteamento, descoberta de topologia de rede, gerenciamento de acesso e segurança em VANETs. Nesta seção serão abordados trabalhos acerca do tema, evidenciando seus principais pontos, sendo descrito no último parágrafo os principais diferenciais em relação à nossa proposta.

Um panorama amplo sobre os trabalhos envolvendo formação de grupos em redes de sensores sem fio, móveis e veiculares é dado por [9]. Tal trabalho descreve os algoritmos desenvolvidos, uma taxonomia base e as principais dificuldades na construção desses tipos de soluções, levando em consideração suas peculiaridades e dificuldades. Entre as dificuldades, ele discute o problema da escolha do líder, afiliação e gerenciamento dos grupos, a validação envolvendo modelos de canais realísticos, assim como a comparação por meio de um benchmark padronizado em um mesmo cenário. Existe ainda a complexidade na comparação entre diferentes trabalhos, oriunda da diversidade de soluções, bem como da utilização de simuladores distintos.

Em [10] é proposta a formação de uma rede construída a partir de grupos de veículos e pedestres. O propósito é levar comunicação a ambientes pós desastres, nos quais o próprio tráfego regular do dia-a-dia não está em seu pleno funcionamento. Existe a figura de um líder para gerenciar a formação do grupo, assim como sua dissolução no caso de haver pouco tráfego associado ao mesmo.

O problema do direito de passagem em cruzamentos é tratado por diversos trabalhos propondo criação de grupos. Em [7] e [8], os autores usam o agrupamento de veículos para simplificar o problema e diminuir a necessidade de troca de mensagens. Em ambas as pesquisas, tem-se o agrupamento de veículos em uma faixa de rolagem, funcionando como um pelotão com seu líder. Assim, ao invés de cada veículo disputar o direito de passagem, o líder o faz para que todo o grupo passe junto com ele. Quanto ao tamanho dos grupos, [8] usa um elemento de infraestrutura externo aos veículos, em combinação com redes neuro-fuzzy, para definir a quantidade de veículos em cada grupo, e também arbitrar direitos de passagem.

Em [14], é apresentado um algoritmo de agrupamento em redes de sensores móveis. Os autores propõem que o líder de um grupo seja o sensor que primeiro se declarar como tal. Já [15] apresenta uma solução que admite a seleção de múltiplos líderes, adotando uma forma de Multihoming, uma vez que o trabalho é voltado para roteamento em VANETs. Os autores apresentam um algoritmo em que todos os veículos se declaram como líderes e, com a recepção de beacons e cálculos de métricas, vão desistindo de tal papel e se tornando membros.

Nosso trabalho se diferencia das abordagens descritas na literatura em dois aspectos fundamentais. $\mathrm{O}$ primeiro é o fato de que ele propõe um protocolo distribuído com o propósito de que cada veículo esteja sempre fazendo parte de um grupo. $\mathrm{O}$ objetivo principal é facilitar a troca rápida de mensagens entre os veículos, reduzindo o tempo necessário para formação de grupos e otimizando o fluxo de informações entre os membros. $\mathrm{O}$ segundo aspecto é a validação da metodologia usando ferramentas de simulação alimentadas por dados reais de tráfego. Para isso, fazemos uso de ferramentas conhecidas na literatura, que são os simuladores VSimRTI [12] e SUMO [16]. Os dados de tráfego são coletados da página oficial da Companhia de Engenharia de Tráfego do Rio de Janeiro (CET-Rio) [13]. Nós descrevemos uma abordagem prática sobre como utilizar dados estatísticos agrupados (fluxo de veículos/dia) para compor um cenário de simulação realístico usando a Distribuição de Poisson [8]. Tal aspecto do trabalho também constitui uma contribuição relevante, dada a dificuldade em reproduzir condições de tráfego reais em ferramentas de simulação [11]. Vale salientar ainda que nossa abordagem se assemelha à proposta de [14] ao desenvolver uma forma de seleção de líder baseada em um backoff aleatório para quem se declarará como membro, tornando um outro veículo líder do grupo; e também pela ausência de métricas diretamente relacionadas ao movimento dos elementos para a seleção do líder, de modo a tornar a formação dos grupos mais célere. Todavia, nossa abordagem é centrada em redes veiculares, em contraste com o trabalho citado, que se baseia em redes de sensores. Nossa proposta também guarda semelhanças com [15], uma vez que todos os veículos iniciam o algoritmo tentando liderar um grupo e, conforme o algoritmo evolui, podem ceder esse papel e se tornar membros.

\section{Metodologia de Agrupamento de Veículos}

Do mesmo modo como pessoas presenciam fatos no cotidiano de suas vidas e guardam em suas memórias versões dos acontecimentos segundo a sua ótica, veículos inteligentes podem guardar em suas On Board Units (OBUs) dados acerca de ocorrências do dia a dia. $\mathrm{O}$ uso inteligente desses dados requer mecanismos eficientes em termos de possibilitar a troca de mensagens entre os integrantes. Para tal, se faz necessária uma abordagem de agrupamento de veículos que favoreça o desenvolvimento de aplicações distribuídas.

A metodologia proposta por este trabalho leva em consideração a construção de um protocolo distribuído eficiente de troca de mensagens entre veículos próximos. Tal comunicação se dá no intuito de possibilitar que veículos se organizem rapidamente em grupos. Um grupo é definido como um conjunto de veículos inteligentes composto por um líder e um ou mais membros, que juntos formam uma associação, sendo cada um deles um nó. O protocolo proposto se aplica a redes $\mathrm{V} 2 \mathrm{X}$. O comportamento dinâmico do protocolo é 


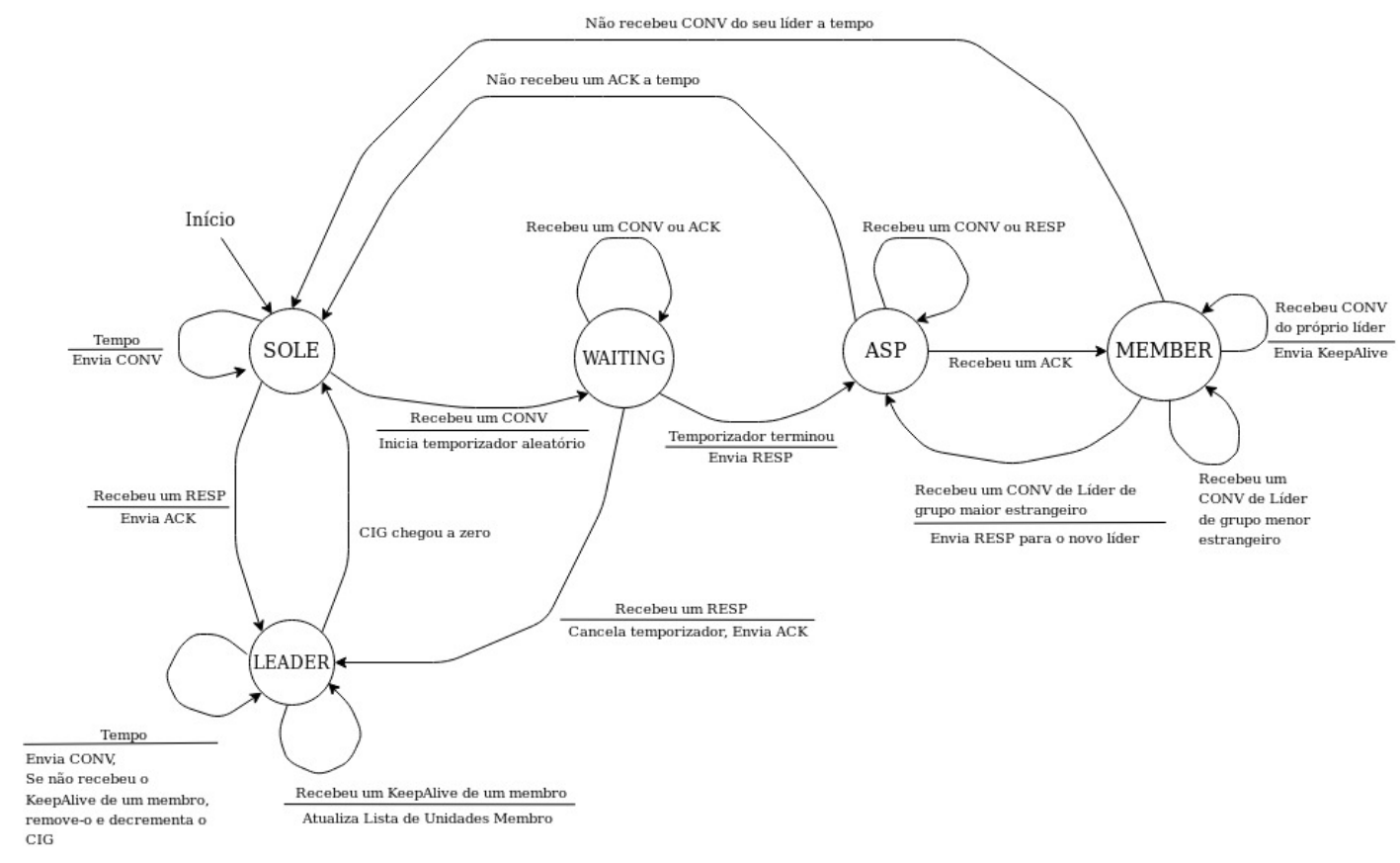

Figura 1. Máquina de estados de formação de grupos

descrito por uma máquina de estados (figura 1), de maneira a facilitar o entendimento de cada etapa da formação dos grupos.

As mensagens indicadas nas transições da máquina de estados apresentada na figura 1 são divididas em quatro tipos: convite (CONV), resposta (RESP), acknowledgement (ACK) e KeepAlive. A figura 2 representa o formato das mensagens no que tange a camada de aplicação, se dividindo em uma estrutura principal comum a todas as mensagens, e dois payloads referentes à CONV e ACK. Cabe ressaltar que as mensagens RESP e KeepAlive não possuem payload específico, sendo diferenciadas das demais apenas pelo campo Tipo da Mensagem na estrutura principal.

Quanto aos campos presentes nas mensagens, destaca-se a funcionalidade do campo Tipo de Mensagem de um byte, o qual efetivamente indica o tipo da mensagem em questão. Outro campo importante é o Número de Identificação do Veículo (NIV), composto por dezessete bytes representando a numeração comumente conhecida como número do chassi do veículo. Além destes, destacam-se o campo de Uso Futuro que poderá conter dados sobre o NIV subsequente e o Padding para preenchimento das mensagens. Por fim, o payload de mensagens do tipo $\mathrm{ACK}$, possui uma região opcional para carregar outras informações acerca do grupo ao qual o membro está sendo adicionado.

\section{A. Construção de Grupos}

A construção de um grupo se dá através do seguinte protocolo. Todas as On Board Units (OBUs) presentes em veículos e possíveis RSUs de sinalizações ao longo da via iniciam suas atividades no estado sozinho (SOLE) e enviam continuamente mensagens do tipo CONV para a formação de um grupo. Tais mensagens são enviadas em modo broadcast para as demais unidades ao alcance de suas comunicações. Quando um veículo recebe um CONV de outra unidade para aderir ou formar um grupo, ele imediatamente pára de realizar o broadcast de seus CONVs, passa ao estado de aguardando (WAITING) e inicia um temporizador aleatório para o envio de uma mensagem de RESP à unidade origem. Quando tal temporizador expira e não foi recebida nenhuma resposta de seus CONVs, a unidade envia uma mensagem RESP à unidade origem, indicando a sua intenção de aderir ao seu grupo, de modo que passa ao estado de aspirante à membro (ASP).

A unidade que tiver um de seus CONVs aceito por pelo menos uma das demais, ou seja, que recebeu uma mensagem do tipo RESP, passará ao estado de líder de grupo (LEADER) e adicionará o novo membro à sua Lista de Unidades Membro (LUM). Neste momento, o LEADER envia uma mensagem do tipo ACK ao novo membro, e torna-se responsável pela manutenção do grupo. As unidades em estado ASP (i.e., aspirantes a membros) que recebem a mensagem ACK passam ao estado de membros do grupo (MEMBER). Cabe ressaltar que líderes de grupos, diferentemente de membros, não param de enviar mensagens CONVs e que tais mensagens carregam consigo a LUM do líder e um Contador de Integrantes no Grupo (CIG). Desse modo, um líder sempre está tentando trazer mais membros para o seu grupo no intuito de fazer com que as informações cheguem ao maior número de unidades possível.

Uma vez construído um grupo, passa-se à fase de manutenção do mesmo, de modo que líderes e membros sempre tenham conhecimento acerca da dissolução do grupo devido ao afastamento das unidades. Nosso protocolo propõe que mensagens do tipo CONV, que são continuamente enviadas em modo broadcast pela unidade líder de um grupo, 

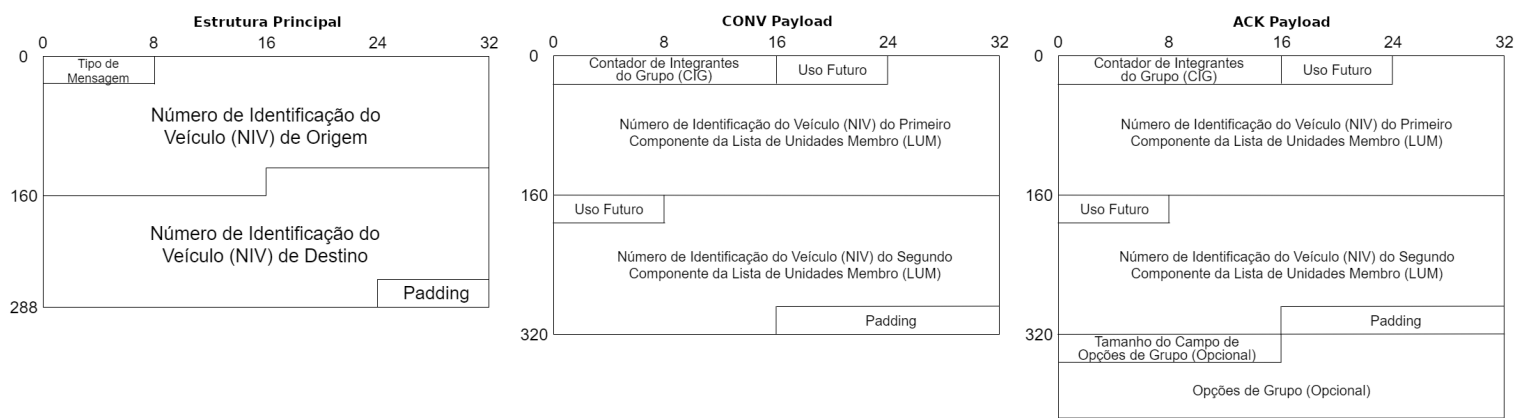

Figura 2. Estrutura principal das mensagens e payloads

sirva para indicar a presença do mesmo para seus membros. Da mesma maneira, uma unidade membro que receba uma mensagem do tipo CONV de seu próprio líder deve responder com uma nova mensagem do tipo KeepAlive, demonstrando ao líder sua presença e que ainda faz parte do grupo. Com isso, todos os integrantes da rede sabem quais veículos do grupo se encontram próximos.

\section{B. Destruição de Grupos}

No momento que uma unidade se distancia fisicamente das demais, saindo do raio de comunicação do líder, ela pára de receber mensagens CONV deste. Nesse momento, devido à ausência de um líder, a unidade percebe que não integra mais o grupo atual. Logo, esta retorna ao estado de SOLE, retomando o envio de suas mensagens do tipo CONV para a formação de novos grupos. Para o líder que perdeu contato com o membro e não recebe mais as mensagens KeepAlive do mesmo, este passa a considerar que o membro não faz mais parte do grupo e deve ser retirado de sua LUM, decrementando o CIG. Quando o CIG de um líder chega a zero, este percebe que não possui mais membros no grupo, passando ao estado SOLE e retomando o início do protocolo.

\section{Junção de Grupos}

Considerando o momento em que um grupo de unidades se encontra com outro já formado previamente, deve haver uma política de junção (merge) entre os dois, com a formação de um único grupo maior. Levando em consideração que, quando um líder envia suas mensagens do tipo CONV, também são encaminhados sua LUM e seu CIG, um membro de um grupo que receba uma CONV do líder de outro grupo terá de decidir a qual formação irá pertencer, baseado nos dados disponíveis. Ou seja, como um membro recebe a todo momento CONVs de seu líder com a LUM e o CIG de seu grupo, pode-se comparar tais dados com aqueles recebidos juntamente com o CONV do líder estrangeiro. Desse modo, o membro decide pelo grupo de maior tamanho. Caso o grupo que convidou seja menor ou igual ao que pertence, ele se mantém no que se encontra. Caso o convite seja de um grupo maior, ele passa ao novo e não responde mais ao seu antigo líder. Conforme um membro muda de grupo, fazendo parte de outro cujo líder estrangeiro possui uma maior quantidade de integrantes, um líder detentor de um grupo menor pode ter seu grupo desfeito pelo processo. Logo, tem-se que um grupo maior pode vir a absorver completamente um menor, uma vez que o líder do grupo pequeno poderá ficar sem membros, retornando ao estado SOLE e aceitando novos convites.

\section{EXPERIMENTOS}

Esta seção avalia o desempenho e os resultados do protocolo proposto, usando uma simulação computacional com dados reais de tráfego. Para tal, foi adotado o modelo de canal Static Rtx [9], no qual todos os veículos, dentro de um raio determinado pela potência do transmissor, recebem a mensagem. Foi utilizado o simulador VSimRTI, que consiste em um framework composto de um conjunto de outros simuladores, possibilitando avaliações de comunicações de rede, dentro de um cenário de tráfego de veículos. Para a reprodução de comunicações $V 2 X$ foi utilizado o simulador Simple Network Simulator (SNS) com alcance radio de 50m, e para a movimentação e simulação de tráfego, o Simulation of Urban Mobility (SUMO).

\section{A. Estratégia de Simulação}

Para a implementação das mensagens e pilhas de protocolos na simulação, foi utilizada a classe V2XMessage provida pelo próprio simulador VSimRTI. Quanto ao envio das mensagens, este foi realizado com métodos próprios da classe abstrata VehicleOperatingSystem, de modo que ambas as classes podem ser consultadas na Application Programming Interface (API) própria do simulador. ${ }^{1}$ Esta implementação teve como foco o desenvolvimento da camada de aplicação, deixando as demais camadas da pilha de protocolos sob responsabilidade do próprio simulador utilizado, segundo o trabalho de [12]. Métricas relacionadas a overhead de canal não foram consideradas neste experimento. Todos os códigos desenvolvidos e utilizados nestas simulações se encontram disponíveis para consulta e reprodução, assim como um trecho gravado em vídeo ${ }^{2}$.

Para a modelagem dos fluxos de veículos e mapas reais de tráfego foram realizadas buscas em centros de engenharia

\footnotetext{
${ }^{1}$ www.dcaiti.tu-berlin.de/research/simulation/download/get/VSimRTI-APIDocumentation-19.1.zip

${ }^{2}$ Código fonte e material de apoio em https://github.com/arvieira/AgrupamentoDeVeiculosInteligentes.
} 
Tabela I

DAdOS SOBRE TRÁFEGo DE VEÍ́CULOS NO BAIRRO DE IPANEMA

\begin{tabular}{|l|l|l|l|}
\hline Id & Rua & Referência & $\begin{array}{l}\text { Veículos por } \\
\text { dia (útil) }\end{array}$ \\
\hline 1 & $\begin{array}{l}\text { Av. Vieira } \\
\text { Souto }\end{array}$ & $\begin{array}{l}\text { Altura da Rua } \\
\text { Maria Quitéria }\end{array}$ & 48.358 \\
\hline 2 & $\begin{array}{l}\text { Rua Visconde } \\
\text { de Pirajá }\end{array}$ & $\begin{array}{l}\text { Altura da Rua } \\
\text { Joana Angélica }\end{array}$ & 16.220 \\
\hline
\end{tabular}

de tráfego das principais cidades do Brasil. Optou-se por utilizar dados da cidade do Rio de Janeiro [13] referentes a uma região de tráfego balanceado, que se comporta como um Grid, semelhante ao projeto urbanístico realizado na cidade de Manhattan [17]. A fonte de dados utilizada disponibiliza apenas a média diária de passagem de veículos por ponto considerado. As características procuradas foram encontradas no bairro de Ipanema. Assim, foi selecionada a região limitada acima pela Rua Visconde de Pirajá, e abaixo pela Avenida Vieira Souto, incluindo as duas vias que compõem a mesma; e lateralmente, na esquerda pela Rua Maria Quitéria, e na direita pela Rua Joana Angélica. Obteve-se um mapa em formato de grid com dois pontos de medição de dados de tráfego. Os dados referentes à região selecionada são exibidos na tabela I.

Após definir a região para simulação, foram estimados os fluxos das vias com base no número de faixas de rolamento, a partir das interseções que possuem dados de tráfego contidos na tabela I. A figura 3 mostra os valores retirados da tabela I dentro de caixas vermelhas, e os valores estimados com base no número de faixas de vias em balões nas cores amarela para fluxos entrantes e azul para aqueles que saem da interseção. O fluxo de 48.358 contido na primeira linha da tabela I representa a média diária de veículos que passam pela referida interseção. Logo, o fluxo que entra no cruzamento, assim como o que sai do mesmo, devem possuir valor igual ao contido na linha 1 da referida tabela. O fluxo entrante se divide entre a Rua Maria Quitéria com 2 faixas, a Av. Vieira Souto sentido Leblon com 3 faixas e a Av. Vieira Souto sentido Arpoador com 3 faixas. O total de faixas entrante é de 8 , de modo que dividindo o valor total do fluxo entrante pelo número de faixas temos que cada uma possui um fluxo de $48358 / 8=6044,75$. O valor obtido pode ser aplicado para cada uma das vias entrantes, resultando nos balões de cor amarela presentes na figura 3. O mesmo procedimento foi aplicado para o fluxo que sai desta interseção, resultando nos valores contidos nos balões azuis.

$\mathrm{O}$ processo descrito anteriormente também foi aplicado para o segundo cruzamento, cujos dados estão disponíveis na segunda linha da tabela I. O resultado final das estimativas de tráfego nas vias, segundo o número de faixas e os dados obtidos em [13], estão disponíveis na figura 3. A mesma figura também mostra as rotas de veículos traçadas para a realização da simulação, as quais respeitam os valores de fluxo diários calculados para cada via. As rotas indicadas, juntamente com suas identificações, cores e valores de fluxo de veículos, encontram-se na tabela II. Cabe ainda ressaltar que, devido a Rota $F$ (em rosa) não influenciar nos valores de fluxos correspondentes aos dados disponíveis em [13], esta pode
Tabela II

ROTAS PROPOSTAS E VALORES DE FLUXO DIÁRIO DE VEÍ́CULOS.

\begin{tabular}{|l|l|l|}
\hline Id & Cor & Valor de Fluxo \\
\hline A & Vermelha & 6044,75 \\
\hline B & Laranja & 6044,75 \\
\hline C & Verde Escuro & 2448,25 \\
\hline D & Amarelo & 4835,5 \\
\hline E & Verde Claro & 1652,5 \\
\hline F & Rosa & Qualquer valor \\
\hline G & Marrom & 18134,25 \\
\hline H & Azul Escuro & 8463,25 \\
\hline I & Cinza & 4835.5 \\
\hline J & Roxo & 4835,5 \\
\hline K & Azul Claro & 2448,25 \\
\hline
\end{tabular}

receber qualquer valor de fluxo, de modo que foi estipulado um fluxo médio de 3600 veículos por dia para viabilizar a simulação, mantendo a validade das demais informações.

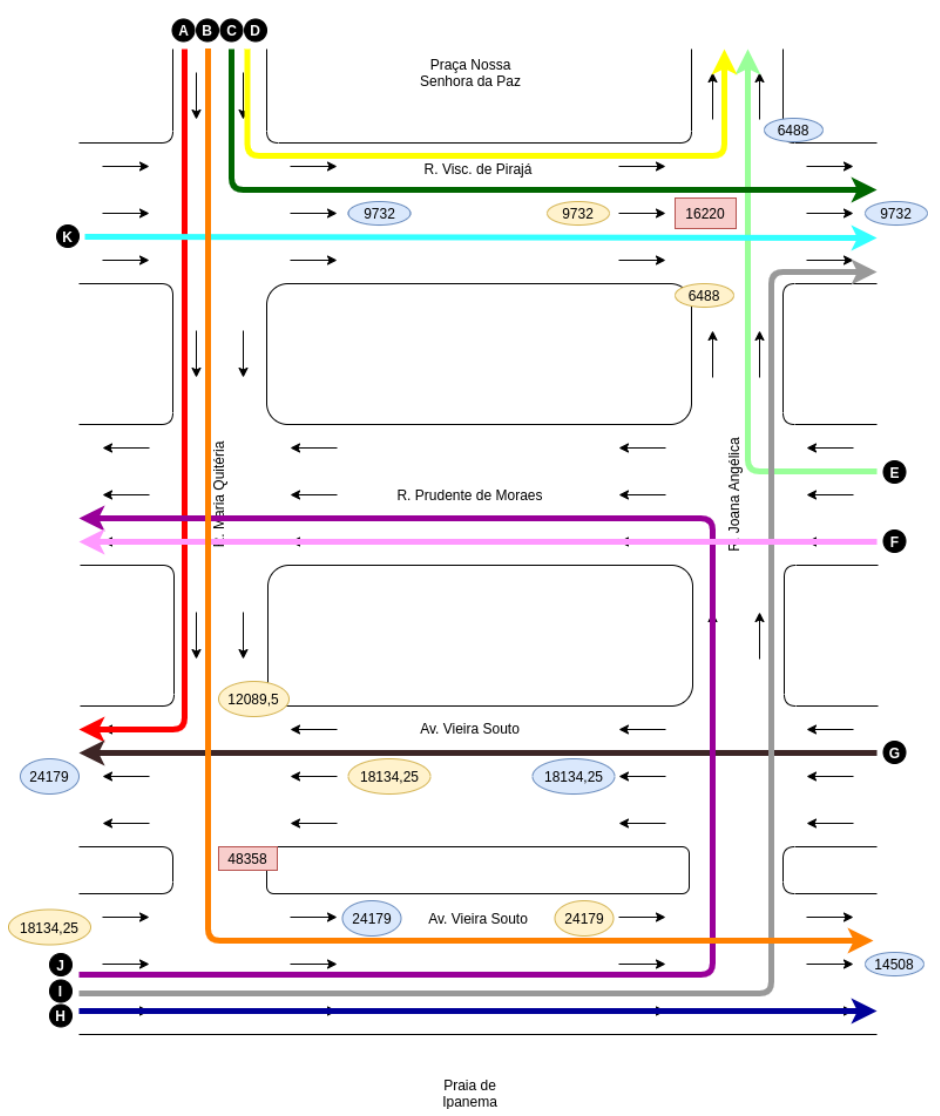

Figura 3. Mapa utilizado para simulação com valores calculados e rotas.

\section{B. Cenários}

Foram realizadas duas simulações com o mapa e rotas descritos na seção anterior. Os valores de fluxo de veículos foram inseridos no mapa segundo uma Distribuição de Poisson, semelhante à utilizada por [8], com taxa igual aos valores contidos na tabela II. Na primeira simulação, foi incluído em cada veículo uma versão do protocolo com a possibilidade de realizar a operação de merge descrita na seção III-C. Na 
segunda, cada veículo recebeu uma versão do protocolo sem a possibilidade de realizar tal operação.

Foi realizada uma simulação usando o VSimRTI, com fluxo de valor equivalente à média diária, e duração equivalente a 24 horas de cada um dos cenários. Não foram consideradas variações noturnas e de horários de pico. Como resultado, foram obtidos arquivos de registros de $\log s$ de funcionamento para cada um dos veículos inseridos no mapa durante a simulação. Para a análise dos dados obtidos, foi construída uma aplicação que realiza a leitura e extração das informações de interesse.

\section{Análise de Métricas da Simulação}

Para análise dos cenários de simulação descritos previamente, foram adotadas as métricas de Tamanho Máximo Atingido, Duração em Segundos dos grupos formados, e o Percentual de Tempo de Agrupamento do veículo. Estas métricas são relevantes porque descrevem como o protocolo se comporta em termos de constituir uma base para o funcionamento de novas aplicações que demandem rapidez e uma taxa específica de formação de grupos, bem como períodos de duração específicos para os mesmos.

Como pré-processamento ao cálculo das métricas, foram removidos da análise os grupos criados dentro do mapa e destruídos fora dele. Isso é necessário pelo fato de que não é possível determinar com precisão o período de duração desses grupos. Pode-se inferir que o mesmo se assemelha aos maiores períodos encontrados para os grupos que são destruídos ainda durante simulação. Todavia, a determinação da duração específica desses grupos não pode ser obtida com precisão pelo fato da simulação envolver também condições realísticas de congestionamento, o que pode afetar mais determinadas rotas. Desse modo, foram removidos 11.344 de um total de 48.030 grupos formados dos resultados da simulação com a funcionalidade de merge, o que representa uma eliminação de 23,62\%. Quanto aos resultados da simulação sem tal funcionalidade, foram removidos 17.311 de um total de 25.052 grupos formados, representando uma remoção de $69,10 \%$. Os efeitos desses percentuais são discutidos mais a frente.

A síntese das métricas obtidas é exibida em formato de histograma nas figuras 4, 5 e 6, com uma linha de quantitativo cumulativo para as duas primeiras. $\mathrm{O}$ gráfico à esquerda da figura 4 remete ao funcionamento do protocolo contendo a funcionalidade de merge, enquanto o gráfico à direita exibe dados relativos ao funcionamento do protocolo sem a funcionalidade de merge. Em ambos os gráficos, o eixo x aponta o tamanho máximo atingido pelos grupos, enquanto os eixos y apresentam à esquerda (em barras verticais) a frequência de grupos que atingiram esses tamanhos e à direita a porcentagem relacionada à curva cumulativa (descrita em linha pontuada). A figura 5 mantém as mesmas características descritas para a figura 4. Todavia, ela corresponde à métrica de duração em segundos dos grupos. A distribuição de frequência mostrada no histograma agrupa as medidas de duração em intervalos de 8 segundos. A figura 6 exibe a razão dada pelo tempo que o veículo permaneceu pertencendo a um grupo, em relação ao tempo total que este participou da simulação. O eixo horizontal está dividido em intervalos de $5 \%$, quanto que o vertical exibe a frequência de veículos que obtiveram um valor naquele intervalo.

É importante observar que na figura 5, o gráfico referente ao funcionamento do protocolo sem a funcionalidade de merge apresenta uma pequena anormalidade quando comparado aos demais. Entendemos que as mesmas podem ser oriundas da possibilidade de grupos terminarem antes do esperado, por terem parte de seus membros saindo das fronteiras do mapa antes de seu líder. Tais ocorrências induzem o término precoce do grupo. Para uma melhor compreensão dessa dinâmica, são apresentados dois gráficos do tipo Dot Map, representando as coordenadas geográficas do líder nos momentos de criação e destruição dos respectivos grupos. A concentração de dados em um mesmo ponto acaba por determinar a intensidade da plotagem, refletindo proporcionalmente a quantidade de grupos criados ou destruídos naquela região.

A figura 7 mostra que há mais criações de grupos quando se aplica a funcionalidade de merge e que tais eventos se concentram na entrada dos veículos no mapa e em cruzamentos. Tal tendência é oriunda da aglomeração de veículos ocasionada pelas características particulares de tais regiões. Quanto a criação de grupos sem o merge, percebe-se que são criados muito menos grupos, e fica evidente a relação com a entrada dos veículos no mapa e a aglomeração de veículos em cruzamentos com semáforos. Os gráficos da figura 8, por sua vez, representam eventos de destruições de grupos. Notase novamente que, quando se utiliza o merge, a destruição de grupos ocorre predominantemente em cruzamentos com semáforos, onde grupos maiores passam por grupos menores e causam sua destruição. Sem a funcionalidade de merge, há menos destruição de grupos, de modo que estas se concentram nas retas, devido ao distanciamento dos veículos em função da diferença de velocidade e aceleração; e nas regiões de fronteira do mapa, devido a saída dos membros do grupo antes do líder.

\section{Discussões}

Com base nos resultados da simulação apresentados na subseção anterior, pode-se afirmar que o protocolo proposto gera grupos de veículos maiores com a utilização da funcionalidade de merge. No entanto, tais grupos duram menos do que aqueles produzidos sem essa funcionalidade. Assim, o uso ou não da funcionalidade de junção de grupos (merge) deve ser avaliado de acordo com as características da aplicação que se deseja desenvolver. Cabe ressaltar que os limites do mapa de simulação influenciam os resultados apresentados. A saída parcial de membros ou do líder de um grupo acaba por causar sua destruição precoce. Este comportamento é observado na figura 5. A distribuição de frequência obtida sem a funcionalidade de merge se distancia da distribuição exponencial recorrente nos demais gráficos. Nesse caso, há a ocorrência de picos de duração, os quais poderiam ser diluídos para grupos de durações maiores caso o mapa não fosse limitado. 

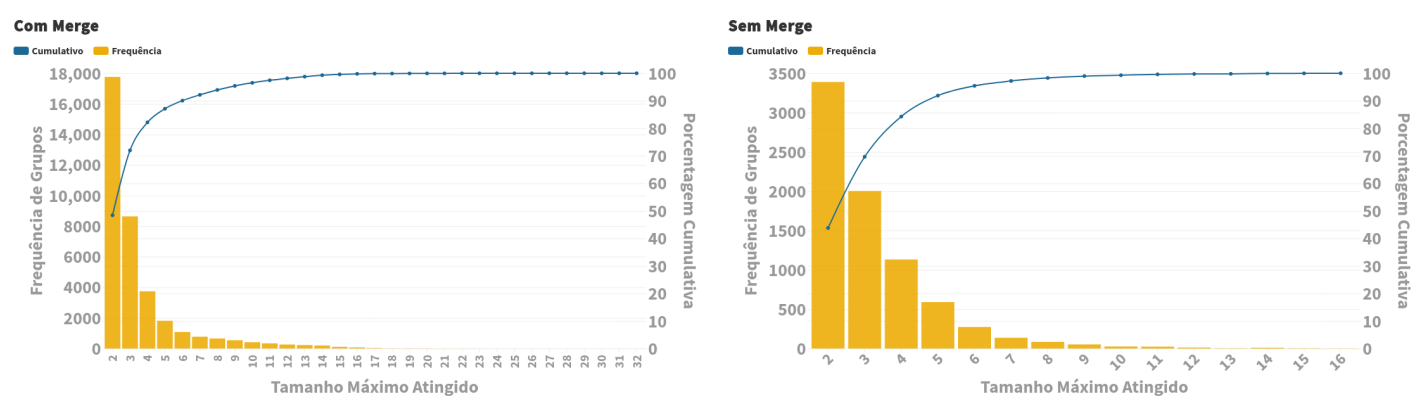

Figura 4. Histogramas de Tamanhos Máximo Atingidos, com e sem merge.
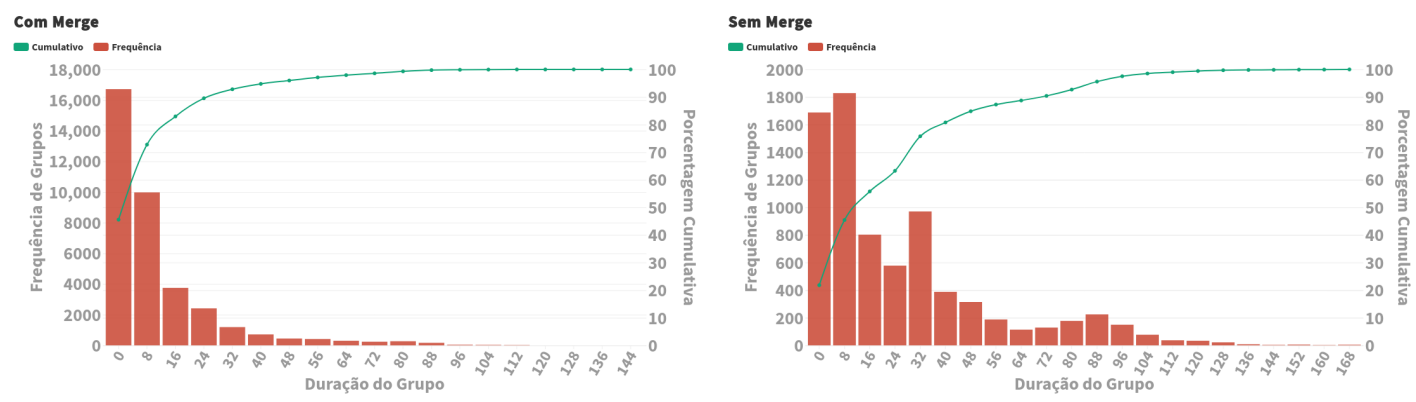

Figura 5. Histogramas de Durações em Segundos com e sem merge.
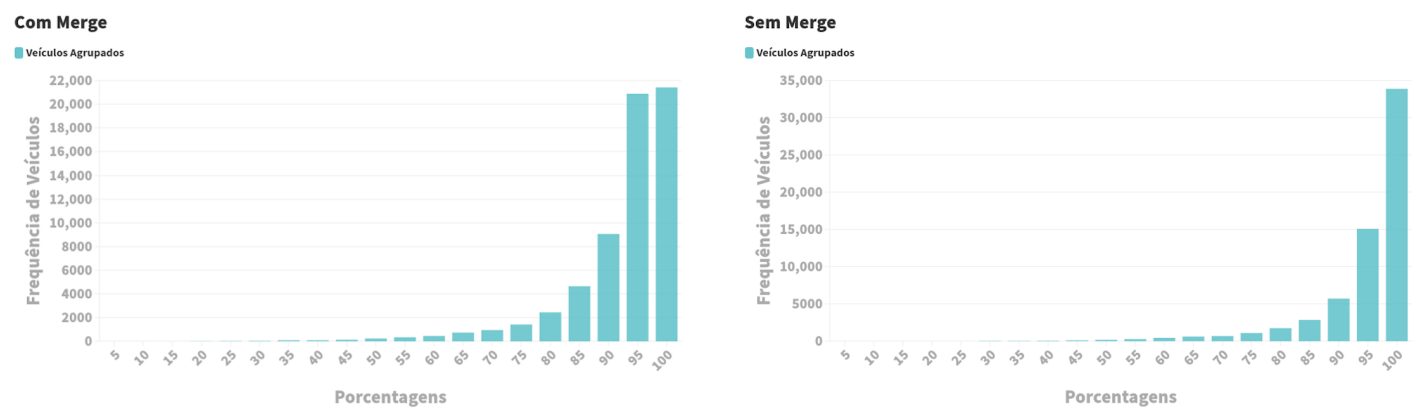

Figura 6. Histogramas de Percentual de Tempo Agrupado por veículo, com e sem merge.

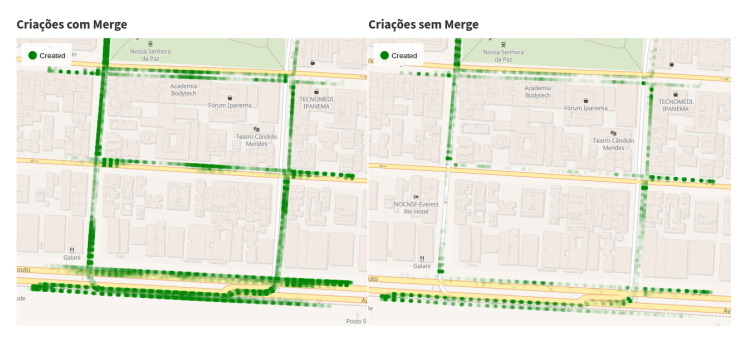

Figura 7. Dot Map de Criações de Grupos com e sem merge

Quanto a velocidade de formação de grupos, foi utilizada a métrica de tempo agrupado em relação ao tempo total que o veículo participou da simulação. Esta métrica foi sumarizada na figura 6 , a qual mostra uma grande concentração acima de $90 \%$ com e sem a utilização da funcionalidade merge. Esta distribuição obtida, possui uma média de $92,15 \%$ e $89,99 \%$

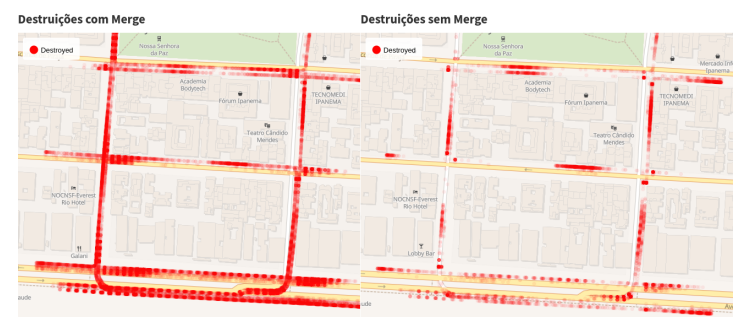

Figura 8. Dot Map de Destruições de Grupos com e sem merge

de tempo agrupado com e sem merge, respectivamente. Desse modo, as formações, dissoluções e reformações de grupo atendem à rapidez projetada.

Por fim, a análise dos Dot Maps (figuras 7 e 8) indica uma predominância de criação de grupos nos pontos de inserção de veículos, uma vez que trata-se de uma simulação em 
mapa limitado. Também são encontradas concentrações de novos grupos próximo a cruzamentos com semáforos, pois nestes locais ocorrem aglomerações de veículos. Observa-se assim que a funcionalidade de merge propicia uma maior formação de grupos. Em relação à destruição de grupos, a análise revela que a funcionalidade de merge aumenta as ocorrências desse evento. Tal fato se dá devido a um grupo maior causar a destruição de grupos menores, atraindo seus membros. Nesse caso, regiões de aglomerações de veículos (e.g., cruzamentos com semáforos) mostram-se muito mais favoráveis a tais eventos. Outro ponto notável é uma grande quantidade de destruição de grupos em retas que sucedem cruzamentos. Esta característica se dá devido à diferença de aceleração e velocidade na partida dos veículos, o que ocasiona um afastamento entre os membros de um grupo e seu líder e, por conseguinte, a destruição prematura do grupo.

Durante as análises de todas as visualizações em conjunto, assim como da representação gráfica gerada pelo próprio simulador $S U M O$, foi constatada a presença de grupos transientes em cruzamentos quando a funcionalidade de merge está presente. Quando um grupo se encontra parado em um semáforo de um cruzamento e um grupo maior passa por tal região, a funcionalidade de merge é executada, de modo que os membros do grupo menor são atraídos para o grupo maior, o qual possui uma característica de ser apenas transiente. Nesse caso, após o grupo maior deixar o cruzamento, os veículos inicialmente pertencentes ao grupo menor e que foram atraídos para o grupo maior, mas permaneceram parados no semáforo, ficam sem grupo. Isso ocasiona o reinício do protocolo para esses veículos. Nesse momento, diversos pequenos grupos são formados e logo a funcionalidade de merge começa a apresentar seus efeitos, unindo os grupos menores, o que gera diversos grupos transientes com pequenos tamanhos e pequenas durações. Estes vão se juntando até que o sistema chegue a uma condição estável semelhante à inicial, antes da passagem do grupo maior pelo cruzamento.

\section{Conclusões e Trabalhos Futuros}

Neste trabalho foi apresentada um nova metodologia para o agrupamento de veículos inteligentes no tráfego. Esta proposta objetivou a criação de um maior número de grupos em menor tempo, assim como uma maior duração desses grupos, de modo a possibilitar futuras aplicações em redes veiculares. Um exemplo de aplicação é a criação de Event Data Recorders (EDR) baseados em Blockchain, que já se encontram em desenvolvimento pelos autores e deve ser apresentada em trabalhos futuros. O protocolo proposto foi avaliado usando o simulador VSimRTI com dados reais de tráfego disponibilizados pela CET-Rio.

Como trabalhos futuros, serão desenvolvidas simulações com mapas maiores e mais dados reais, além de estudos de estabilidade dos grupos com a funcionalidade merge, e também dos grupos transientes. A ideia é desenvolver técnicas para minimizar problemas causados por esses grupos, levando em consideração um intervalo de tempo até que o grupo sofra merge e a velocidade relativa entre o membro e o líder do grupo maior, conforme sugerido por [9]. A comparação com outros protocolos de criação de grupos também será objeto de trabalhos futuros, devido a complexidade já descrita de se estabelecer comparações com trabalhos prévios. $\mathrm{O}$ estudo de aplicações da proposta, assim como a utilização de outras métricas em cenários mais complexos, também serão objeto de próximas pesquisas.

\section{REFERÊNCIAS}

[1] J. E. Siegel, D. C. Erb, and S. E. Sarma, "A survey of the connected vehicle landscape-architectures, enabling technologies, applications, and development areas," IEEE Transactions on Intelligent Transportation Systems, vol. 19, no. 8, pp. 2391-2406, 2017.

[2] L. L. Bello, S. Mubeen, S. Saponara, R. Mariani, and U. D. Bordoloi, "Guest editorial embedded and networked systems for intelligent vehicles and robots," IEEE Transactions on Industrial Informatics, vol. 15, no. 2, pp. 1035-1037, 2019.

[3] V. D. Sagar and T. Nanjundeswaraswamy, "Artificial intelligence in autonomous vehicles-a literature review," -Manager's Journal on Future Engineering and Technology, vol. 14, no. 3, p. 56, 2019.

[4] O. Kayis and T. Acarman, "Clustering formation for inter-vehicle communication," in 2007 IEEE Intelligent Transportation Systems Conference, Sep. 2007, pp. 636-641.

[5] C. Frese, T. Batz, M. Wieser, and J. Beyerer, "Life cycle management for cooperative groups of cognitive automobiles in a distributed environment," in 2008 IEEE Intelligent Vehicles Symposium, June 2008, pp. $1125-1130$.

[6] A. Marjovi, M. Vasic, J. Lemaitre, and A. Martinoli, "Distributed graphbased convoy control for networked intelligent vehicles," in 2015 IEEE Intelligent Vehicles Symposium (IV), June 2015, pp. 138-143.

[7] W. Ni, W. Wu, and $\mathrm{K}$. $\mathrm{Li}$, "A message efficient intersection control algorithm for intelligent transportation in smart cities," Future Generation Computer Systems, vol. 76, pp. 339 - 349, 2017. [Online]. Available: http://www.sciencedirect.com/science/article/ pii/S0167739X16304794

[8] J. Cheng, W. Wu, J. Cao, and K. Li, "Fuzzy group-based intersection control via vehicular networks for smart transportations," IEEE Transactions on Industrial Informatics, vol. 13, no. 2, pp. 751-758, April 2017.

[9] C. Cooper, D. Franklin, M. Ros, F. Safaei, and M. Abolhasan, "A comparative survey of vanet clustering techniques," IEEE Communications Surveys \& Tutorials, vol. PP, pp. 1-1, 092016.

[10] H. Nishiyama, T. Ngo, S. Oiyama, and N. Kato, "Relay by smart device: Innovative communications for efficient information sharing among vehicles and pedestrians," IEEE Vehicular Technology Magazine, vol. 10, no. 4, pp. 54-62, Dec 2015.

[11] B. Bloessl and A. O'Driscoll, "A case for good defaults: Pitfalls in vanet physical layer simulations," in 2019 Wireless Days (WD). IEEE, 2019, pp. 1-6.

[12] B. Hilt, M. Berbineau, A. Vinel, and A. Pirovano, Simulation of Convergent Networks for Intelligent Transport Systems with VSimRTI. Wiley, 2017, ch. 1, pp. 1-28. [Online]. Available: https://ieeexplore.iee.org/document/8045560

[13] CET-RJ, "SINFRERJ estatísticas de volume de tráfego," https://www.sinfrerj.org.br/site/index.php/textos/pagina/152/ Estatisticas-de-volume-de-trafego, 2019, accessed: 2019-12-06.

[14] Taek Jin Kwon, M. Gerla, V. K. Varma, M. Barton, and T. R. Hsing, "Efficient flooding with passive clustering-an overhead-free selective forward mechanism for ad hoc/sensor networks," Proceedings of the IEEE, vol. 91, no. 8, pp. 1210-1220, 2003.

[15] S. Vodopivec, J. Bester, and A. Kos, "A multihoming clustering algorithm for vehicular ad hoc networks," International Journal of Distributed Sensor Networks, vol. 2014, pp. 1-8, 032014.

[16] D. Krajzewicz, J. Erdmann, M. Behrisch, and L. Bieker, "Recent development and applications of SUMO - Simulation of Urban MObility," International Journal On Advances in Systems and Measurements, vol. 5, no. 3\&4, pp. 128-138, December 2012. [Online]. Available: http://elib.dlr.de/80483/

[17] R. S. Rose-Redwood, "Rationalizing the landscape: superimposing the grid upon the island of manhattan," Ph.D. dissertation, Pennsylvania State University, 2002. 